\title{
Solar Neutron Telescope Count Peaks Not Due to Gamma Rays
}

\author{
Edward Bramlitt ${ }^{1}$ \\ Bramlitt Consulting Services \\ 8813 Camino Osito NE, Albuquerque, NM 87111 USA \\ E-mail: ebramlittemsn.com
}

\begin{abstract}
The 7 March 2011 count history for the solar neutron telescope (SNT) at Mt. Sierra Negra shows two peaks of equal intensity one hour apart. Several reasons have been given for the earlier peak to be due to solar gamma rays, and it was the first experimental data of solar gamma rays reaching the ground. The significant result is solar gamma rays must have exposed aircraft flying at low latitudes. The Fermi satellite, in low-earth orbit, detected a solar gamma ray event that day, but gamma rays did not arrive at Fermi until after the first SNT count peak, and the maximum flux at Fermi was less than count rates for the two peaks. Further, the second SNT peak occurred when the Sun was closer to the SNT at Mt. Mauna Kea, and no peak was reported for that SNT. Weather data rule out thunderstorms as a cause of gamma rays at the SNT, and the SNT energy threshold excludes counts by radioactivity. In conclusion, solar gamma rays were not counted at the ground, so there is no proof they reached flight levels.
\end{abstract}

35th International Cosmic Ray Conference - ICRC2017

10-20 July, 2017

Bexco, Busan, Korea

${ }^{1}$ Speaker 


\section{Introduction}

Commercial aircraft are routinely exposed to elevated levels of galactic cosmic rays (GCR), and Earth's magnetic field causes the radiation dose rate to be highest on polar-route flights. A solar proton event (SPE) can add dose, and the USA Federal Aviation Administration (FAA) requires airlines flying polar routes to mitigate exposures during solar flare activity [1]. A solar gamma ray event (SGE) can also add dose, but to low-latitude flights as gamma rays are not affected by magnetic fields.

The solar neutron telescope (SNT) at Mt. Sierra Negra, Mexico, had a count peak on 7 March 2011 from solar gamma rays; the first ever detection of them at ground level [2]. The SNT responds to gamma rays and neutrons, but it was gamma rays that day as (1) count ratios differed from ones in 2005 when the SNT detected neutrons, (2) an M3.7 solar flare near noon at the SNT caused an SGE detected by the Fermi satellite in low-earth orbit, and (3) Monte Carlo calculations support the cause being $\mathrm{GeV}$ gamma rays.

Bramlitt and Shonka [3] refer to the SNT event as a new concern for air travel radiation safety as it means elevated doses to aircraft flying at low latitudes. The highest SGE doses will be to west-bound daylight flights near the subsolar point (SP). Although SP are tropical, solar gamma rays encounter aircraft cruising at long horizontal distances from SP. For example, there is only a $50 \%$ increase in air shielding for planes cruising at $11.8 \mathrm{~km}$ altitudes and 5,300 km from the SP, and that shielding benefit is less than being atop Mt. Everest.

Aircraft may have been exposed to solar gamma rays for an extended time as the count record at [2] shows two similar peaks an hour apart, although [2] only acknowledges the first peak. That possibility warranted study of [2] and the associated SGE. The study results are reported here by Section 2 with characteristics of the count peaks and SP at the time of peaks, Section 3 detailing the SGE time, energy, and intensity at the Fermi satellite, and Section 4 with a summary of factors pertinent to the SNT and SGE. Section 5 concludes the cause of SNT count peak is unknown but not gamma rays, solar gamma rays have not yet been detected at ground level, and the SGE may not have caused any dose at flight levels.

\section{The SNT Count Peaks}

Figure 1 displays a digitized version of the SNT count histogram at [2] but with their incorrect MST (Mountain Standard Time) label changed to Central Standard Time (CST). Western Mexico had MST, but the east and SNT had CST in March 2011 but starting on April 3 they had Central Daylight Time (CDT). Reference [2] claims the count peak centered at 13:55 is due to solar gamma rays from an M3.7 flare, but it is silent about the peak at 14:53 and its cause. Here the 13:55 peak is named Peak 1 and the 14:53 peak is Peak 2. Universal time (UT) is CST +6 and CDT +5 . Thus, Peaks 1 and 2 were at 19:55 and 20:53 UT. That day also the GOES 15 satellite registered solar X-ray irradiance increase leading to a dominant peak near 20:10. The peak began with a long gradual irradiance increase that fit a polynomial trendline with $\mathrm{R}^{2}=0.996$. The line inflection point at 19:33 is deemed the peak start time. Weaker X ray peaks surrounded the main peak at 17:16, 18:15, 18:24, 20:37, 21:50, and 22:19. 


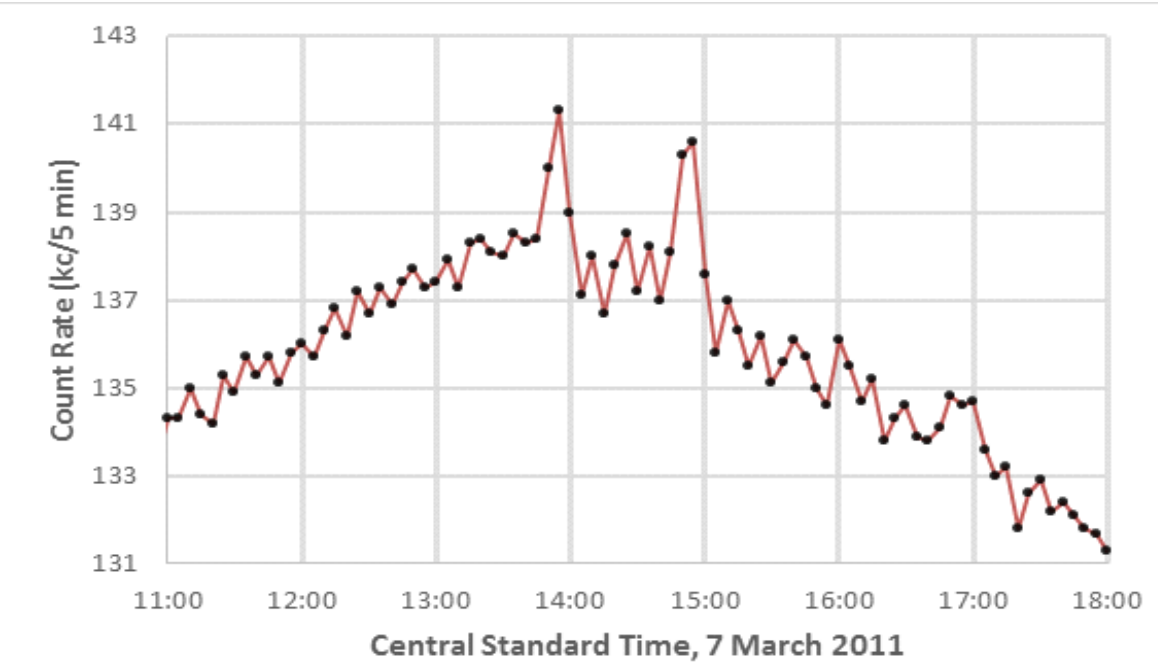

Figure 1. The SNT 7 March 2011 count record. Data points were read from the histogram at [2]. Here the peak at 13:55 is called Peak 1 and the peak at 14:53 is Peak 2.

The Peak 1 net count rate by [2] is $380 \pm 43$ counts per minute (cpm). Peak 1 spans three 5min gross counts totaling 141,300 . The SNT background due to cosmic and terrestrial sources is not public, so here it is estimated by a line from the average of two counts before and after the peak. The Peak 1 net rate is $430 \pm 61 \mathrm{cpm}$, with uncertainty the square root of gross plus background rates with each divided by its count time. Peak 2 spans four 5 -min counts totaling 140,600 , and its net rate by the routine for Peak 1 is $430 \pm 53 \mathrm{cpm}$.

The intensity of solar neutrons and gamma rays on Earth is greatest at SP, so knowing SP relative to the SNT is important for evaluating SNT counting. The NOAA Solar Calculator is an online resource which makes determining SP easy. Give the Calculator SNT coordinates, date, time, and time zone, and it returns declination, equation of time (EOT), solar noon, azimuth, and elevation. Declination is SP latitude (SP-lat). SP longitude (SP-lon) is by Equation 1 from [3]:

$$
\text { SP-lon }=(720-\text { EoT }- \text { UT }) / 4
$$

where UT is expressed in minutes by (UT hours)(60) + (UT minutes).

Table 1 characterizes Peaks 1 and 2 starting with date/time and net count rate plus $1 \sigma$ uncertainty. Next is SP coordinates at peak times, and then SP location relative to SNT by azimuth, elevation and distance at ground level obtained from the online GPS Latitude and Longitude Distance Calculator. Mass thickness of air between the SNT and Sun is M based on $575 \mathrm{~g} / \mathrm{cm}^{2}$ air pressure [2], and M alt based on $621 \pm 1 \mathrm{~g} / \mathrm{cm}^{2}$ measured at the SNT on 7 March 2011 [4]. The air pressure given at [2] apparently is calculated using $4.6 \mathrm{~km}$ as the elevation of Mt. Sierra Negra and the SNT. 


\begin{tabular}{|c|c|c|c|c|c|c|c|c|c|c|}
\hline $\begin{array}{c}\text { Peak } \\
(\#)\end{array}$ & $\begin{array}{l}\text { Date Time } \\
(\mathrm{m} / \mathrm{d} / \mathrm{y} \mathrm{h}: \mathrm{m})\end{array}$ & $\begin{array}{l}\text { Rate } \\
(\mathrm{cpm})\end{array}$ & $\begin{array}{l}\text { Error } \\
(\mathrm{cpm})\end{array}$ & $\begin{array}{l}\text { SP lat } \\
\text { (deg) }\end{array}$ & $\begin{array}{c}\text { SP lon } \\
\text { (deg) }\end{array}$ & $\begin{array}{c}\text { A } \\
(\mathrm{deg})\end{array}$ & $\begin{array}{c}\mathbf{E} \\
(\mathrm{deg})\end{array}$ & $\begin{array}{c}\mathbf{D} \\
(\mathrm{km})\end{array}$ & $\begin{array}{c}\mathbf{M} \\
\left(\mathrm{g} / \mathrm{cm}^{2}\right)\end{array}$ & $\begin{array}{c}\text { M alt } \\
\left(\mathrm{g} / \mathrm{cm}^{2}\right) \\
\end{array}$ \\
\hline 1 & $3 / 7 / 11$ 19:55 & 430 & 61 & -5.15 & -116 & 219 & 59.6 & 2,517 & 666 & 720 \\
\hline 2 & $3 / 7 / 1120: 53$ & 430 & 53 & -5.15 & -131 & 237 & 49.4 & 3,959 & 758 & 818 \\
\hline
\end{tabular}

Table 1. SNT count peaks by date/time and their net count rates with $1 \sigma$ uncertainty. The subsolar point (SP) at date/time was at SP-lat and SP-lon degrees. From the SNT the SP was at azimuth (A), elevation (E), and ground distance (D) with air mass thickness (M) separating the SNT and SP. The M are for SNT air pressure $575 \mathrm{~g} / \mathrm{cm}^{2}$ [2]; the $\mathrm{M}$ alt are for $621 \pm 1 \mathrm{~g} / \mathrm{cm}^{2}$ measured at the SNT on 7 March 2011 [4].

Solar noon at the SNT on 7 March 2011 was 18:40:19, so the count peaks were registered in the afternoon. The SP was southwest of the SNT for both count peaks, elevation decreased with time, and azimuth and distance increased. Peaks 1 and 2 had equal count rates, but Peak 2 occurred when the SP was 1,612 km further west and closer to the Mt. Mauna Kea SNT.

\section{Solar Gamma Rays Over Earth}

The Fermi satellite orbits Earth with a $0.95 \mathrm{~m}^{2}$ large area telescope (LAT) to count gamma rays $>100 \mathrm{MeV}$. LAT data is available from the Fermi solar flare $\mathrm{X}$ ray and gamma ray observations site (hesperia.gsfc.nasa.gov/Fermi solar). LAT Daily Lightcurve and Spectrum files list counts for all minutes and place counts in energy bands. Times for significant solar exposure are at nasa.gov/lat/lat_solar_exposure_times.txt with LAT effective area for the exposures.

Figure 2 plots starting times for solar exposures vs. count rates and rates divided by LAT effective area which averaged $0.26 \pm 0.06 \mathrm{~m}^{2}$. Each rate is total count for the orbit divided by the count duration. Error bars are $1 \sigma$ based on counts only. Exposure times preceded count times by $3.6 \pm 1.5 \mathrm{~min}$. Average time between the 16 orbits was $2.6 \mathrm{hr}$, and count durations were $31 \pm$ $15 \mathrm{~min}$. The five high rates are SGE; othrs are gamma ray background which averaged $0.53 \pm$ $0.13 \mathrm{cpm}$ and $2.33 \pm 0.94 \mathrm{cpm} / \mathrm{m}^{2}$. The background rate distribution is Poisson.

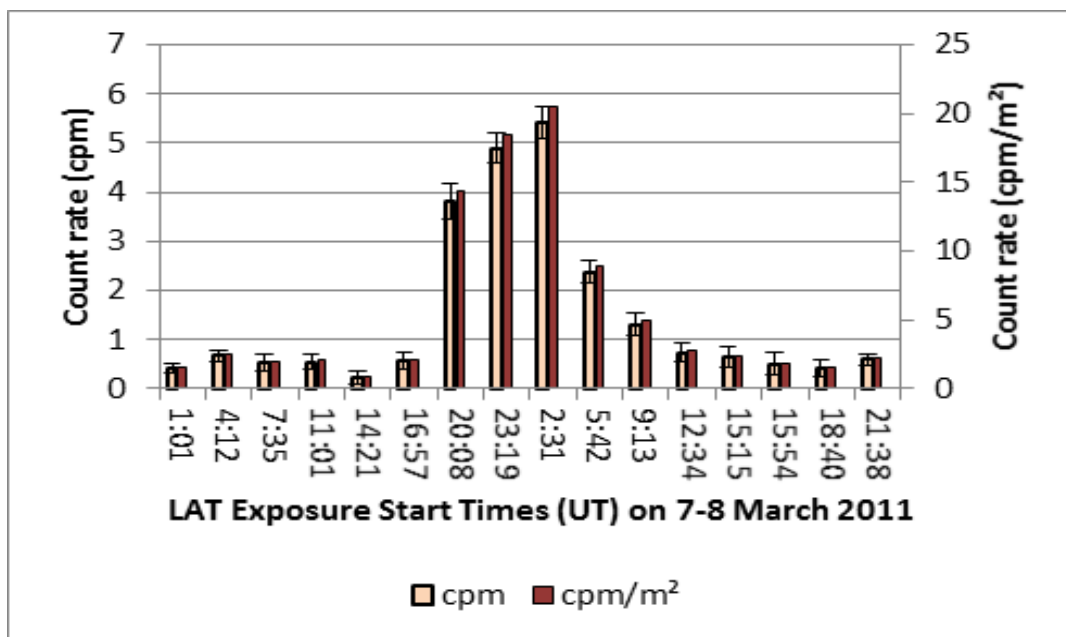

Figure 2. Fermi LAT counting of solar gamma rays $>100 \mathrm{MeV}$ on 7-8 March 2011. Count rates (yellow) have $1 \sigma$ error bars and, after division by LAT effective area, display in red. Exposure starts precede count starts by a few minutes. An SGE caused the five highest rates; the other rates are gamma ray background.

The first SGE count by Fermi was from 20:10 to 20:39 on 7 March 2011. All 29 1-min counts were $>0$ and totaled 122 . LAT spectral data show $98 \%$ of those counts were $<800 \mathrm{MeV}$. Three counts fell in the 0.8 to $1.5 \mathrm{GeV}$ range at 20:22 and 20:23, but no counts were by higher 
energy gamma rays. The background was more energetic as $10 \%$ of its counts were in the 0.8 to $1.5 \mathrm{GeV}$ range, $3.8 \%$ at 1.5 to $4 \mathrm{GeV}$, and $0.63 \%$ at 4 to $10 \mathrm{GeV}$.

The 122 count trend is upward with time, but with much scatter. Trendlines were determined for counts summed at 3, 4, 5, and 6 min intervals vs. minutes after 20:10. All were linear, but the 5 -min sums had the best fit with $\mathrm{R}^{2}=0.95$. Figure 3 plots the 5 -min sums net of background with $1 \sigma$ error bars based on counts. The intercept at zero count rate is -5.2 min indicating SGE gamma rays began arriving over Earth on 7 March 2011 around 20:05. Apparently, Fermi happened to resume solar exposure near the start of SGE gamma rays arriving at Earth.

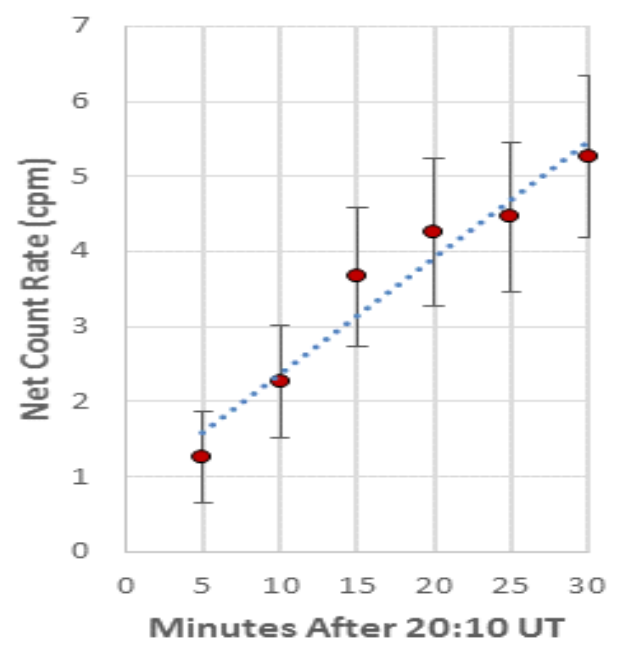

Figure 3. Fermi LAT counting of SGE gamma rays began at 20:10 and lasted $29 \mathrm{~min}$. The plot points are consecutive 5-min count sums net of background with $1 \sigma$ error bars based on counts. The linear trendline quality is good with $\mathrm{R}^{2}=0.95$, and the positive slope is reasonable near an SGE start. The trendline intercept at $0 \mathrm{cpm}$ is $-5.2 \mathrm{~min}$, suggesting SGE gamma rays first arrived over Earth around 20:05.

Fermi has orbited Earth 50,000 times in nine years during which solar viewing time was 29.8 $\pm 14.6 \mathrm{~min}$ and LAT effective area was $0.19 \pm 0.12 \mathrm{~m}^{2}$. (The orbits of Figure 2 are thus ordinary.) The Fermi website converts LAT counts to flux, and it maintains a LAT significant events list (SEL) of all orbits with significant flux. The SEL gives date, start time, count duration, and flux with a measure of significance. There have been 54 significant counts, but counts occurring close in time are probably due to a single solar event. Accordingly, Fermi has monitored 26 SGE since the first significant flux from 20:10 to 20:39 on 7 March 2011.

Figure 4 displays that first significant flux with the SNT Peak 1 and Peak 2 count rates. Flux by the SEL is $2.23 \mathrm{E}-05 \gamma / \mathrm{cm}^{2} / \mathrm{s}$. It is $268 \gamma / 4 \mathrm{~m}^{2} / 5 \mathrm{~min}$ for comparison with SNT whose detector area is $4 \mathrm{~m}^{2}$ [2]. That flux was received during the 29-min exposure, so for Figure 4 it has been scaled by the 5-min LAT counts. Thus, flux for the six red columns sums to 268 . The intensity of SNT counts at ground level is greater than the intensity of gamma rays above the atmosphere. 


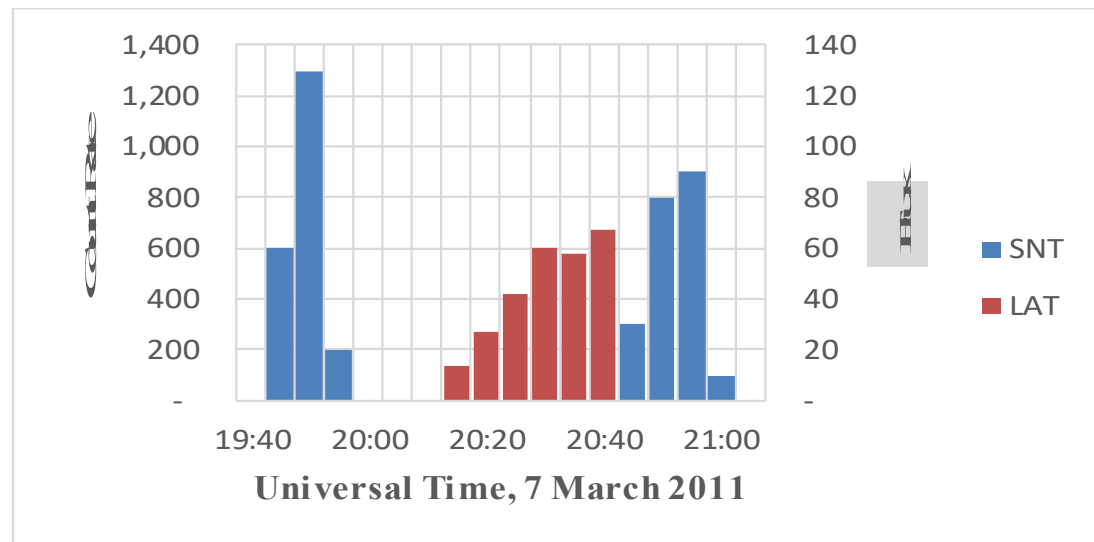

Figure 4. Gren columns to the left are SNT Peak 1 and to the right are Peak 2. Red columns express LAT counts as flux with area adjusted to the SNT detector area. All columns are 5-min wide.

4. Discussion Solar gamma rays did not begin to arrive at Earth until after Peak 1. The Fermi satellite counted SGE gamma rays on five orbits. The first orbit had statistically significant flux by 122 counts from 20:10 to 20:39, counts at 5-min intervals increased linearly with time, and the line intercept indicates SGE gamma rays began arriving over Earth at 20:05. Peak 1 began at 19:50, reached maximum at 19:55, and returned to background at 20:00.

Solar gamma rays at Fermi lacked the energy to cause SNT counts. Reference [2] says the path length for $84.4 \mathrm{MeV}$ gamma rays in air is $37.7 \mathrm{~g} / \mathrm{cm}^{2}$ and air over the SNT was $575 \mathrm{~g} / \mathrm{cm}^{2}$. That air shielding is 15 mean free paths, so solar gamma rays of that energy will never reach the SNT. But, Monte Carlo calculations referred to at [2] say gamma rays $>3 \mathrm{GeV}$ reach it and are detected with $100 \%$ efficiency, and $1 \mathrm{GeV}$ gamma rays reach it with $20 \%$ efficiency. The Fermi LAT places each gamma ray detection in one of eight energy bands. Over the total 3.4 hours of SGE counts there were 7 gamma rays $>1.5 \mathrm{GeV}$, and for the total 4.9 hours of background counts there were also 7 $>1.5 \mathrm{GeV}$. Peak 1 and Peak 2 each had 2,100 counts, or about 100 conts when adjusted to the LAT effective area for SGE counts. The few $\mathrm{GeV}$ gamma rays in 3.4 hours at Fermi were not sufficient to cause 100 counts in 15 or 20 min of SNT counts.

Solar gamma rays at Fermi lacked the intensity to cause SNT counts. By [2] the Peak 1 count rate was $380 \mathrm{cpm}$ and the SNT detection area is $4 \mathrm{~m}^{2}$. The SGE maximum flux at Fermi was 3.7E$05 \gamma / \mathrm{cm}^{2} / \mathrm{s}$ or $89 \mathrm{\gamma pm} / 4 \mathrm{~m}^{2}$. Intensity was lower above the air than counts under the air.

No count peak was reported for the Mt. Mauna Kea SNT even though it was closest to the Peak 2 SP, and the SNT network is maintained with solar cycle 24 expectations presented in 2009 [5]. The Peak $2 \mathrm{SP}$ was at longitude $131.5^{\circ} \mathrm{W}$ which is $34^{\circ}$ west of Mt. Sierra Negra (4.58 $\mathrm{km}$ a.s.1.) and $24^{\circ}$ east of Mt. Mauna Kea (4.21 km a.s.1.). The SNT-SP distances were 3,960 and $3,110 \mathrm{~km}$, respectively. A cause more than the M3.7 flare is needed to account for Peak 2.

Thunderstorms generate gamma rays in a flash called a terrestrial gamma flash (TGF). There are $\sim 1,100$ TGF worldwide each day at latitudes encompassing the SNT. But, there was no thunderstorm near the SNT during Peaks 1 and 2, according to archived data from a weather station located $150 \mathrm{~m}$ from the SNT [6]. 
The count peak distributions appear more normal than skewed, as for counts of a long-lived radioactive source with constant geometry and shielding. But, the SNT detection threshold is $30 \mathrm{MeV}$ [2] and too high for radioactivity. For example, the decay energy of ${ }^{60} \mathrm{Co}$ is $2.5 \mathrm{MeV}$.

Neutrons did not cause Peak 1 for some reasons given at [2]. Additionally, the Mexico City neutron monitor $(\mathrm{NM})$ had no count peak but a constant rate $(231 \pm 1.5 \mathrm{c} / 5 \mathrm{~min})$ for five hours around Peaks 1 and 2. In 2005 it had a peak in time with a solar neutron peak at the SNT. The NM to SP azimuth was $131^{\circ}$ in 2005 and $183^{\circ}$ in 2011 at 19:55, and ground distances differed by only 7\%. The solar flare was stronger in 2005, but the SP was near due south in 2011.

The USA Space Weather Prediction Center reported an SPE starting at 1:05 on 8 March 2011. Peaks 1 and 2 were earlier and thus not associated with radiation from that SPE.

Say an SNT count peak was expected due to the M3.7 flare near noon at the SNT, but say the SNT count record had no peak. Let some counts be added during CDT to make a peak at 14:55 $\mathrm{CDT}=19: 55$ UT. But wait; the record is in CST, so repeat the addition for a peak at 13:55 CST $=19: 55$ UT. The enhanced record has peaks one hour apart, but the expectation is met.

\section{Conclusion}

The SNT count record for 7 March 2011 shows two similar peaks one hour apart with intensities too large to be statistical anomalies. The earlier peak is claimed at [2] to be due to solar gamma rays associated with an M3.7 flare, but, that is not possible as solar gamma rays did not begin arriving over Earth until after the peak. No cause is given at [2] for Peak 2, but it must be more intense than for Peak 1 as the SP had moved far to the west. Solar gamma rays are excluded as the cause of either peak because they lacked the necessary energy and intensity to cause the counts. They are excluded as cause of Peak 2 also because no peak was reported for the closest SNT, the one at Mt. Mauna Kea, USA. The count peaks were not caused by TGF gamma rays as there was no thunderstorm to create TGF, nor were they due to gamma rays from radioactivity because of the SNT high-energy detection threshold. Solar neutrons are excluded for causing count peaks by some reasons given at [2], plus the NM at Mexico City was flatline. There was no timely SPE to cause the peaks. The cause of SNT count peaks is unknown, but it was not gamma rays. Solar gamma rays have not yet been detected at ground level, and there is doubt of them exposing aircraft flying at low latitudes. This work shows SP characteristics are valuable to interpreting SNT counting. The NOAA Solar Calculator and Equation (1) here make determining those characteristics easy.

\section{Acknowledgements}

This work was unfunded. It used products from the Fermi solar flare observations facility funded by the Fermi Guest Investigator program, and received valuable help promptly from Kim Tolbert at the facility. It used Mexico City NM data from the NMDB database (www.nmdb.eu) founded under the European Union's FP7 program (contract no. 21307), and that data is kindly provided to the NMDB by the Cosmic Ray Group, Geophysical Institute, National Autonomous University of Mexico (UNAM), Mexico. I thank Alberto Carramiñana and Daniel Ferrusca for retrieving Mt. Sierra Negra weather data, Jorge Torres (Chief Meteorologist at a station in New Mexico) for opinions on that data, and E. Frank Bramlitt for digitizing charts and providing computer assistance to his visually impaired father. 


\section{References}

[1] FAA. Extended operations (ETOPS and Polar Operations), Advisory Circ 120-42B, 2008.

[2] Y. Muraki, J. F. Valdes-Galicia, J. F. Gonzalez, K. Koga, H. Matsumoto, et al., A possible detection of solar gamma-rays by the ground level detector, 33rd ICRC, Rio de Janeiro 2013.

[3] E. T. Bramlitt and J. J. Shonka, Radiation exposure of aviation crew members and cancer, Health Phys. 108(1): 76-81, 2015.

[4] A. Carramiñana, Mt. Sierra Negra weather info, email to E. Bramlitt, 12 May 2016.

[5] Y. Matsubara, Y. Muraki, T. Sako, Y Itow, T. Sakai, et al., Status of the world-wide network of solar neutron telescopes in solar cycle 24, 31 ${ }^{\text {st }}$ ICRC, Lodz 2009.

[6] D. Tierney, M. S. Briggs, G. Fitzpatrick, V. L. Chaplin, S. Foley, et al., Fluence distribution of terrestrial gamma ray flashes observed by the Fermi gamma-ray burst monitor, J. Geophys. Res. Space Phys. 118, 6644-6650, 2013. 